\title{
Manuscrits d'auteur, norme linguistique et « critique des variantes » dans la tradition littéraire italienne
}

\section{Christian Del Vento}

\section{(2) OpenEdition Journals}

Édition électronique

URL : https://journals.openedition.org/genesis/4410

DOI : 10.4000/genesis.4410

ISSN : 2268-1590

Éditeur :

Presses universitaires de Paris Sorbonne (PUPS), Société internationale de génétique artistique littéraire et scientifique (SIGALES)

\section{Édition imprimée}

Date de publication : 15 décembre 2019

Pagination : 15-29

ISBN : 979-10-231-0650-3

ISSN : $1167-5101$

\section{Référence électronique}

Christian Del Vento, « Manuscrits d'auteur, norme linguistique et « critique des variantes » dans la tradition littéraire italienne », Genesis [En ligne], 49 | 2019, mis en ligne le 01 décembre 2020, consulté le 03 septembre 2021. URL : http://journals.openedition.org/genesis/4410 ; DOI : https://doi.org/ 10.4000/genesis.4410 


\title{
Manuscrits d'auteur, norme linguistique et "critique des variantes" dans la tradition littéraire italienne
}

\author{
Christian Del Vento
}

L e 17 juin 1783, un jeune homme de trente-quatre ans, le comte Vittorio Alfieri, qui est en train d'accomplir un long périple à travers la Péninsule et l'Europe pour promouvoir la toute nouvelle édition de ses tragédies, en laquelle il fonde ses espoirs de gloire littéraire, fait une halte à Ferrare. C'est la quatrième étape d'un véritable «pèlerinage poétique» qui le conduit en autant de lieux que le panthéon littéraire italien compte de grands auteurs : après Ravenne (Dante), Arqua (Pétrarque) et Sorrente (le Tasse), la visite à Ferrare recèle une signification plus profonde. Alfieri ne se limite pas à y visiter «la maison, et la tombe» de l'Arioste, comme il l'écrit dans la première rédaction de son autobiographie, mais il y contemple aussi un objet d'une nature toute particulière : ses manuscrits ${ }^{1}$, que l'on pouvait consulter dans la bibliothèque municipale depuis 1753, avec ceux du Tasse. Ces mêmes manuscrits feront l'objet, cent cinquante ans plus tard, en 1937, de la première édition génétique italienne, peut-être même de la première édition génétique tout court, établie par Santorre Debenedetti2 ${ }^{2}$. Alfieri consulte les célèbres manuscrits du Roland furieux, en y laissant des traces de son passage destinées à devenir elles-mêmes un objet de dévotion, comme en témoignera, cinquante ans plus tard, un voyageur français d'exception, Antoine-Claude Pasquin Valéry :

Alfieri s'inclinant devant ce manuscrit obtint la permission d'y inscrire les mots : Vittorio Alfieri vide e venerò, 18 giugno 1783. Le custode, garçon singulièrement solennel et pathétique, s'exprimant con la cantilena romana, montre même la trace d'une larme versée par Alfieri³.

Une autre trace de cette visite nous intéresse davantage : parmi les livres de l'écrivain qui sont aujourd'hui conservés à la médiathèque Émile Zola de Montpellier, se trouve un exemplaire de l'édition du Roland furieux qu'avait imprimée à Lyon, en 1557, le célèbre typographe Guillaume Rouillé4. C'est un petit in-16 qu'Alfieri avait acheté à Sienne en 1777 et dont il ne se sépara jamais. Relié en maroquin et portant ses initiales, ce livre fait partie des quelques dizaines de volumes que l'auteur emporta avec lui, en août 1792, lorsqu'il s'échappa

1. Il s'agit des manuscrits classés Cl. I, A, It. 1 de la Biblioteca Comunale Ariostea de Ferrare, donnés par deux généreux collectionneurs, Giuseppe Carli, en 1750, et Giovanni Andrea Barotti, en 1769.

2. L. Ariosto, I frammenti autografi dell'Orlando furioso, éd. S. Debenedetti, Turin, Chiantore, 1937, réédités récemment avec une introduction de Cesare Segre (Rome, Edizioni di Storia e Letteratura, 2010). Sur ces manuscrits, sur cette édition, sur sa fortune critique et sur le rôle qu'elle eut dans le débat né autour de la «critique des variantes » et de Gianfranco Contini voir, dans ce même numéro, l'enjeu de Simone Albonico.

3. A. C. Pasquin Valéry, Voyages historiques et littéraires en Italie pendant les années 1826, 1827, 1828; ou L'Indicateur italien, Paris, Crapelet, 1831-1835, vol. II, p. 75. Voir M. Preti, La lacrima di Alfieri. Ariosto e i patrioti, dans I voli dell'Ariosto. L'Orlando furioso e le arti, M. Cogotti, V. Farinelli, M. Preti (dir.), Milan, Officina Libraria, 2016, p. 119-134, notamment p. 119. L'annotation d'Alfieri se lit dans le ms. Cl. I A, It. 1, f. $53 \mathrm{vv}^{\circ}$.

4. Orlando furioso di M. Ludovico Ariosto, diviso in due parti..., Lyon, G. Rouillé, 1557 [Montpellier, Médiathèque centrale Émile Zola, 34629 Rés.].

Les «incunables de la critique génétique» 
précipitamment de Paris en y laissant presque toute sa bibliothèque 5 . Ce n'est pas simplement en raison de son format de poche, facilement transportable, qu'Alfieri sauva ce volume : c'est aussi parce qu'il constituait une relique précieuse de son pèlerinage. Les marges étroites et les «seuils» sont parsemés de notes (fig. 1). Alfieri ne s'était pas limité à y transcrire des vers, voire des strophes entières du poème telles qu'il avait pu les lire à Ferrare sur le «manuscrit de sa main $» 6$; il y note bien d'autres éléments : les strophes modifiées en profondeur par l'auteur du Roland furieux; la présence de ratures et le nombre des campagnes de corrections que l'on peut y distinguer; l'endroit où commence le manuscrit par rapport à l'édition imprimée... En y regardant de plus près, on constate l'intérêt qu'Alfieri porte aux variantes : il consigne ainsi sur le verso de la troisième page de garde antérieure la première ébauche des vers 7-8 de la strophe 79 du chant IX («Ma gli fu dietro Orlando con più fretta, / che non esce da l'arco una saetta» : «Mais Roland l'atteignit plus vite qu'une flèche n'est chassée de l'arc») 7 :

La Stanza 79 dicea

Ma non fù Orlando a seguir lui più tardo

Che dietro al Capriol veloce pardo si stringa il

Che a seguir lepre, o Capriol sia
La Strophe 79 disait

Mais Roland ne fut à le poursuivre plus lent

Que, derrière le Chevreuil, véloce léopard se presse le

Qu'à suivre lièvre ou Chevreuil ne l'est

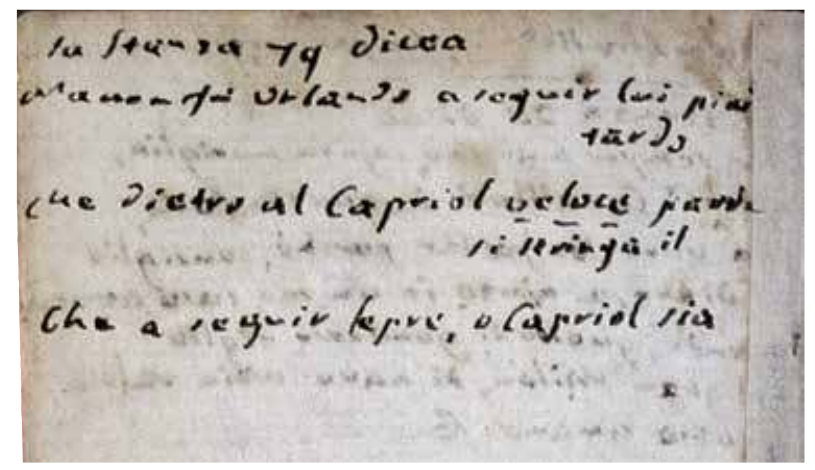

Fig. 1 : Annotations de la main d'Alfieri dans son exemplaire d'Orlando furioso di M. Ludovico Ariosto, diviso in due parti..., Lyon, G. Rouillé, 1557, 3e feuille de garde ant. $\mathrm{v}^{\circ}$.

๑) 2019 Montpellier, Médiathèque centrale Émile Zola, 34629 Rés.

Alfieri reproduit ici la diachronie des corrections : il dispose les variantes en colonne, en regard du mot ou de la portion de vers qui avait fait l'objet d'un changement. Ainsi, on comprend que l'Arioste avait d'abord écrit Che dietro al Capriol veloce pardo («Que, derrière le Chevreuil, véloce léopard»); puis qu'il avait remplacé le mot veloce (souligné par Alfieri) par le syntagme si stringa il («se presse le»); et qu'il avait fini par reformuler le vers tout entier : Che a seguir lepre, o Capriol sia («Qu'à suivre lièvre ou Chevreuil ne l'est»).

5. Sur les vicissitudes des livres d'Alfieri voir C. Del Vento, La biblioteca ritrovata. La prima biblioteca di Vittorio Alfieri, Alessandria, Edizioni dell'Orso, 2019.

6. Orlando Furioso di M. Ludovico Ariosto, diviso in due parti..., op. cit., p. 757.

7. Ibid., p. 197. Nous citons la traduction classique de Francisque Reynard (L'Arioste, Roland furieux, traduction nouvelle par F. Reynard, Paris, A. Lemerre, 1880, t. I, p. 185). Nous traduisons les variantes. 
Pourquoi cet intérêt d'Alfieri pour les variantes ? Et d'où tenait-il ce système de transcription qui le rapproche du généticien moderne ? On trouve une réponse à la première question dans l'édition de 1652 de la Jérusalem délivrée 8 qu'Alfieri avait achetée à Sienne en même temps que son exemplaire de l'Arioste. Sur le verso de la page de garde antérieure de ce petit volume in-32, également relié en maroquin et frappé de ses initiales, Alfieri a collationné les «modifications » de la première et de la dernière strophe du poème, telles qu'il pouvait les lire sur le manuscrit autographe du Tasse (fig. 2) :

Nel manoscritto di Ferrara intero, e postillato di man del Tasso, son varie mutazioni; di cui parte ebbero luogo, parte nò. Serva d'esempio.

Stanza prima.

«Che favorillo il Cielo \& -

«Segni ridusse i suoi \& -

Corresse l'autore peggiorando.

«Che il Ciel li diè favore $\&$ -

«Segni ritenne i suoi \& -

\section{e Stanza ultima}

«Spogliato appena il sanguinoso \&

corresse megliorando come stà ora :

«Non pur deposto il sanguinoso \& -

Sur le manuscrit de Ferrare, complet et annoté de la main du Tasse, apparaissent diverses modifications, dont certaines aboutirent, d'autres non. Par exemple :

Strophe I.

«Que le Ciel le protégea etc. -

«Sous les [saints] étendards il ramena ses etc. -

L'auteur corrigea en empirant.

«Que le Ciel lui fut favorable etc. -

«Sous les [saints] étendards il retint ses etc. -

Et la dernière Strophe

«Aussitôt ôtée la [cape] ensanglantée etc.

il corrigea en l'améliorant, comme on le lit aujourd'hui :

«Sans déposer la [cape] ensanglantée etc. -

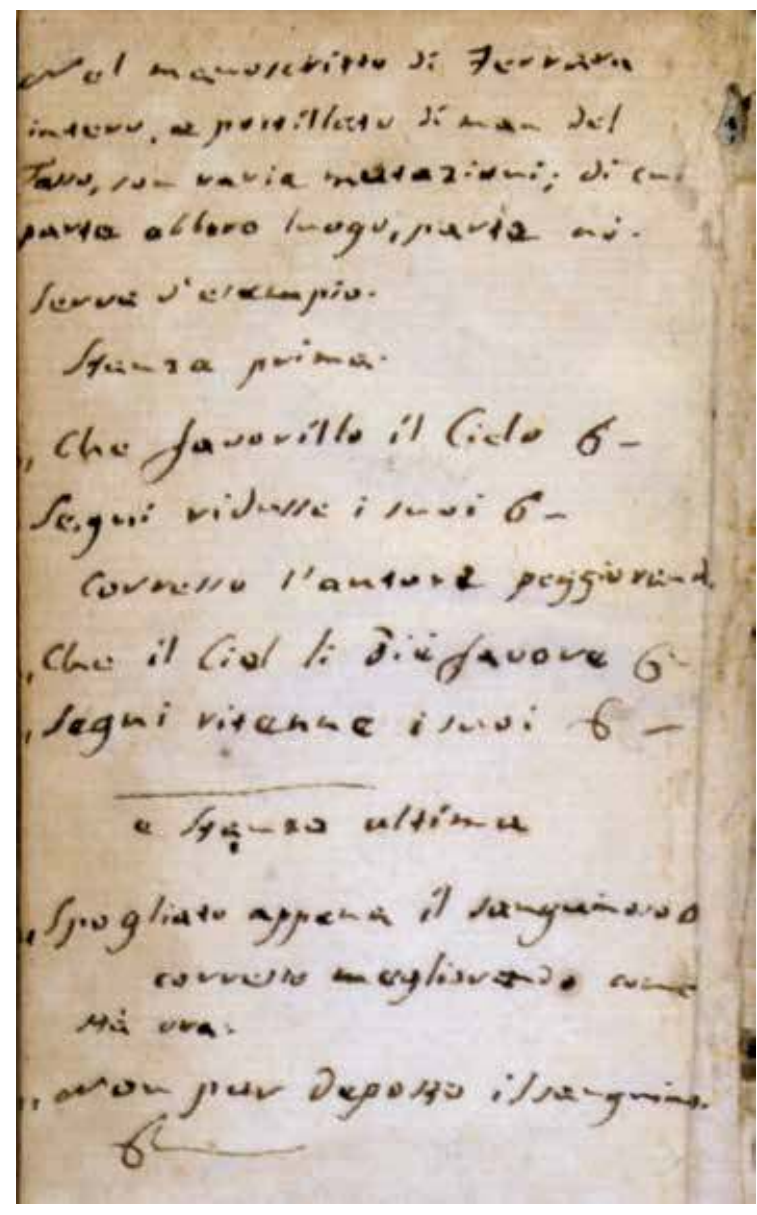

Fig. 2 : Annotations de la main d'Alfieri dans son exemplaire de Il Goffredo del Torquato Tasso, Amsterdam, Combi et la Nouè, 1652, feuille de garde ant. vº (C) 2019 Montpellier, Médiathèque centrale Émile Zola, 34632 Rés.

8. Il Goffredo del Torquato Tasso, Amsterdam, Combi et la Nouè, 1652 [Montpellier, Médiathèque centrale Émile Zola, 34632 Rés.]. Goffredo est le titre sous lequel la Jérusalem délivrée avait paru pour la première fois, à l'été 1580. 
On comprend aisément qu'Alfieri n'explore pas l'atelier du Tasse et de l'Arioste en érudit, pour établir l'édition d'un texte, mais en apprenti. Les manuscrits des deux grands écrivains sont le laboratoire de son apprentissage et l'examen philologique du manuscrit se transforme en démarche poétique.

Alfieri suivait les préconisations d'un des hommes de lettres les plus célèbres de la première moitié du XVIII e siècle, Ludovico Antonio Muratori, qui, dès 1711, avait publié ce qui fut, au siècle des Lumières, l'édition de référence du Chansonnier de Pétrarque. Muratori rappelait dans sa préface :

Ce n'est enfin pas un mince avantage pour les jeunes amateurs de Belles Lettres que de contempler la façon dont les Maîtres talentueux changent, corrigent et améliorent leurs Compositions : et il n'est pas de mets plus doux pour la curiosité des plus savants que d'avoir, en un sens, sous les yeux le même Original que contempla Pétrarque [...] et de pouvoir ici observer quelle était l'Orthographe utilisée en ce temps, et par un Auteur si célèbre, et de quelle obéissance faisaient preuve les fantaisies de son imagination et quelle était l'abondance de pensées et de mots dont se prévalait le Prince de la Poésie lyrique italienne9.

Dans son édition, Muratori avait choisi de reprendre le texte de Pétrarque tel qu'il avait été publié en 1642 par Federico Ubaldini ${ }^{10}$. Ce véritable «incunable de la critique génétique ${ }^{11}$ » reproduisait par des procédés typographiques raffinés ce qu'on appelle aujourd'hui le Codice degli abbozzi (manuscrit Latin 3196 de la Bibliothèque Vaticane), c'est-à-dire les brouillons du Chansonnier, avec toutes les corrections, ratures et variantes que Pétrarque y avait consignées (fig. 3). Dans ce volume, composé de vingt feuillets, étaient conservés de nombreux textes, dont certains sont entrés dans le recueil du Chansonnier, alors que d'autres ont alimenté le corpus des compositions dites «extravagantes». On peut lire en marge de ces textes de nombreuses annotations en latin de la main de Pétrarque, qui témoignent, selon les cas, des circonstances dans lesquelles les poèmes avaient vu le jour, des différentes phases de leur élaboration, des corrections particulières ou, simplement, des passages qu'il souhaitait réviser. Ces textes, qui se présentent tant sous la forme de premiers jets que de copies de travail, voire, parfois, de transcriptions définitives (bien qu'ensuite retouchées par endroits), ont été composés au cours d'une période qui embrasse la vie entière du poète ${ }^{12}$.

9. Le rime di Francesco Petrarca riscontrate co' i testi a penna della libreria Estense, e co' i fragmenti dell'originale d'esso poeta..., Modène, B. Soliani, 1711, p. XIX (nous traduisons).

10. [F. Ubaldini], Le Rime di Messer Francesco Petrarca estratte da un suo originale..., Rome, Grignani, 1642. 11. Selon la définition de C. Segre, «Petrarca e gl'incunaboli della critica genetica», Critica del testo, VII, 1-3, 2003, p. 3-8, notamment p. 3. Voir aussi, du même auteur, «Critique des variantes et critique génétique», Genesis, $\mathrm{n}^{\circ} 7,1995$, p. 29-45, notamment p. 32.

12. Voir L. Paolino, «Dans le laboratoire de Pétrarque. Les variantes d'auteur du Chansonnier et des Triomphes», Arzanà, $\mathrm{n}^{\circ}$ 5, 2000, p. 57-77, notamment p. 62-63, ainsi que F. Petrarca, Il codice degli abbozzi. Edizione e storia del manoscritto Vaticano latino 3196, éd. L. Paolino, Milan-Naples, Ricciardi, 2000. Sur la modernité de l'approche d'Ubaldini, voir P. Italia, «Alle orgini della filologia d'autore. L'edizione del "Codice degli abbozzi" di Federico Ubaldini», dans La filologia in Italia nel Rinascimento, C. Caruso et E. Russo (dir.), Rome, Edizioni di Storia e Letteratura, 2018, p. 379-398. 


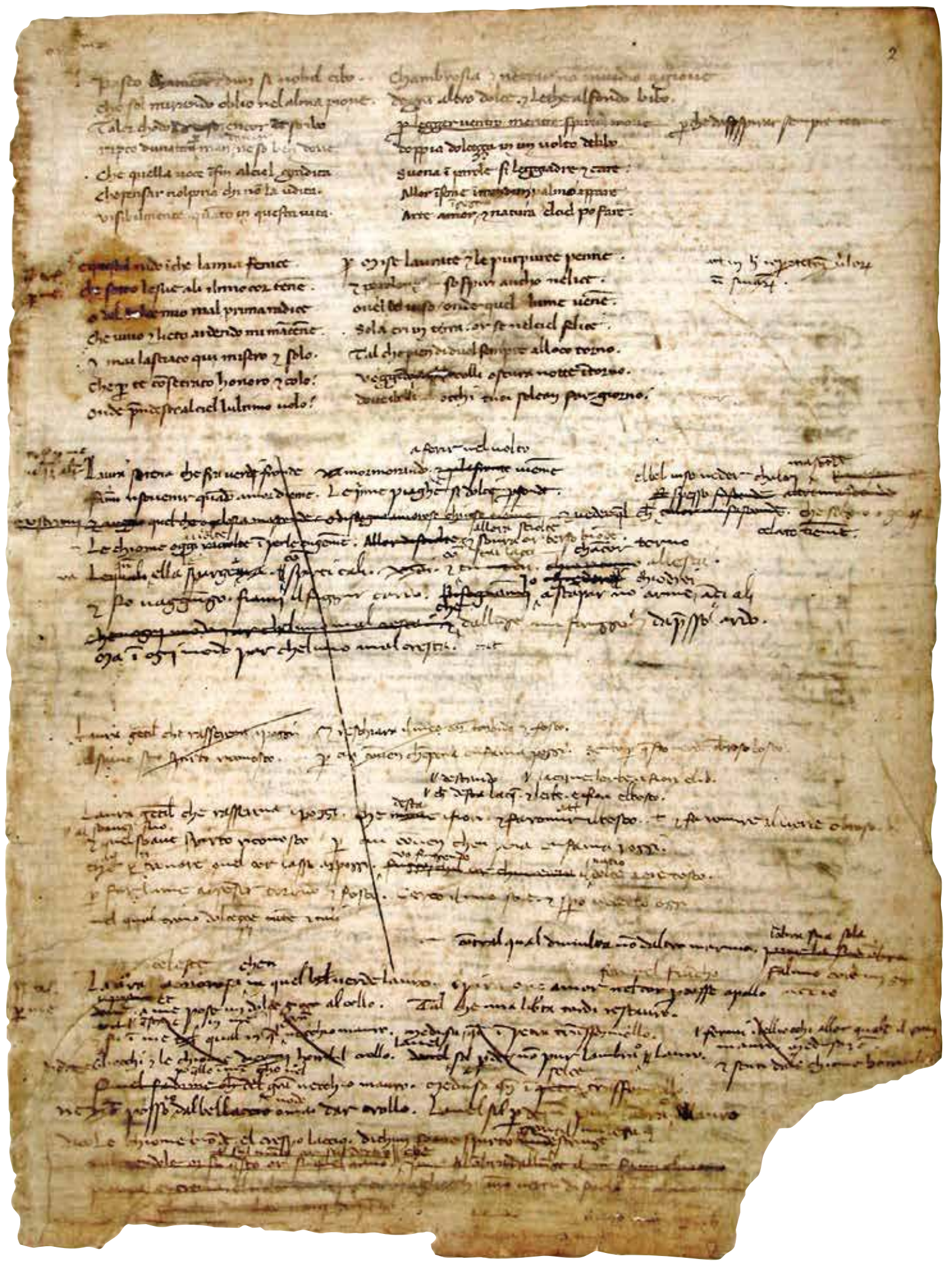

Fig. 3 : Pétrarque, Codice degli abbozzi, Vat. lat. 3196, f. $2 \mathrm{r}^{\circ}$.

(C) 2013, Cité du Vatican, Bibliothèque Apostolique Vaticane 
Dans son introduction, Muratori semble vouloir anticiper les critiques que son choix risquait de susciter, celles-là mêmes qu'allait formuler deux siècles et demi plus tard, en 1947, le champion du néo-idéalisme italien, Benedetto Croce, à l'encontre de Gianfranco Contini ${ }^{13}$ :

Je sais très bien que certains se moqueront d'une telle méticulosité et feront passer l'attention que nous portons à toute ces minuties pour une entreprise pédantesque; et Ubaldini et moi-même pourrons nous estimer heureux si personne ne nous accuse d'être de superstitieux adorateurs de Pétrarque, comme s'il s'agissait de vénérer jusqu' aux embryons de Messire Francesco, et d'accorder trop d'importance à ce que lui-même méprisa et décida d'ensevelir dans l'oubli14.

D'où venait donc cette idée de collationner et de commenter les corrections et les variantes de Pétrarque, mais aussi d'autres écrivains de la tradition littéraire italienne, tels que Boccace, l'Arioste ou le Tasse ? Elle remontait à la Renaissance, et précisément à la première moitié du Xvie siècle. En 1541, l'homme de lettres lucquois Bernardino Daniello s'était aidé des variantes connues des poèmes de Pétrarque pour rédiger un commentaire des sonnets, chansons et Triomphes qui avait rencontré un franc succès. Dans la deuxième édition de son texte, parue en 154915, Daniello décida de consacrer aux variantes une section spécifique afin d'y examiner et de comprendre la logique des «mutationi» introduites par le poète. Le but était éminemment pédagogique. En plein courant pétrarquiste, la connaissance des variantes du Chansonnier et des Triomphes devait aider tous ceux qui voulaient écrire à perfectionner leur technique et leur style en suivant l'exemple du maître, en considérant les erreurs et les corrections qui avaient permis à sa poésie d'atteindre la perfection. Il s'agissait de mesurer, à partir des brouillons de Pétrarque, «l'écart entre la poésie en devenir et la poésie réalisée $16_{»}$. Daniello avait ouvert une voie nouvelle dans l'étude des textes des grands écrivains.

Ce travail de commentaire des manuscrits et des variantes de Pétrarque avait été rendu possible grâce à la concentration entre les mains de deux hauts prélats, Baldassarre Turini (1486-1543) et Pietro Bembo (1470-1547), au début du Xvie siècle, d'une grande partie des archives littéraires du poète. L'intérêt pour les autographes des écrivains, et de Pétrarque en particulier, n'était pas nouveau. L'autographe du Chansonnier avait déjà servi à l'établissement de l'édition de 1472, publiée par Bartolomeo Valdezocco et Martino di Siebeneichen, comme en témoigne le colophon indiquant que le texte fut imprimé ex originali libro,

13. Sur ce débat fondamental, né en 1947, qui orienta en profondeur la critique littéraire italienne de la deuxième moitié du xxe siècle, et sur la position de Gianfranco Contini voir, dans ce même numéro, les enjeux de Simone Albonico et Paola Italia et les références bibliographiques évoquées. Pour un panorama en langue française sur ce débat important, voir M. T. Giaveri, «La critique génétique en Italie : Contini, Croce et 1'“étude des paperasses”», Genesis, $\mathrm{n}^{\circ} 3,1993$, p. 9-29, notamment p. 19-29.

14. Le rime di Francesco Petrarca, op. cit., p. XVIII (nous traduisons).

15. Sonetti canzoni e triomphi di M. Francesco Petrarca, con la spositione di Bernardino Daniello..., Venise, [P. et G. Nicolini da Sabbio], 1549.

16. Voir L. Paolino, art. cit., p. 60, et G. Belloni, Laura tra Petrarca e Bembo. Studi sul commento umanisticorinascimentale al «Canzoniere», Padoue, Antenore, 1992, p. 234. Sur l'étude, au XvI siècle, des variantes dans l'œuvre des grands auteurs de la tradition italienne, voir la synthèse de C. Segre, «Petrarca, Ariosto e la critica delle varianti nel Cinquecento », dans La critica letteraria dal Due al Novecento, P. Orvieto (dir.), Rome, Salerno (Storia della letteratura italiana, 11), 2003, p. 353-367, et, du même auteur, «Critique des variantes et critique génétique», art. cit., p. 32. 